\title{
The Effect of the Improve of Posture by Tilt-table Stepping Robot Rehabilitation on Walking Ability in Cerebral Palsy.
}

\author{
Hyo-Jung Park ${ }^{1}$, Byong-Yong Hwang ${ }^{2}$, In-Tae Ryu ${ }^{* 3}$ \\ ${ }^{1}$ Daejeon Rehabilitation Hospital, \\ ${ }^{2}$ Dept. of Physical Therapy, Yong-in University, \\ ${ }^{*}$ Daejeon Rehabilitation Hospital
}

\begin{abstract}
Purpose The purpose of this study was to investigate changes in standing posture using tilt-table stepping robot rehabilitation, in the context of posture specificity of patients with rigid bilateral cerebral palsy. Methods In the case of an 18-year-old spastic diplegia cerebral palsy patient, Erigo an upright robot rehabilitation exercise-and Bobath-concept general physical therapy were performed for 30 minutes, three times per week, for four weeks from August 16 to September 4, 2018. Coordination of balance and body movement in static and dynamic sitting postures was evaluated with the Korean Trunk Impairment Scale (K-TIS), and Postural Assessment Scale for Stroke-Trunk Control (PASS) and 10-meter walking test and GAITRite were used to evaluate walking ability. The static pressure of the foot was measured using a BIORescue. To assess postural changes before and after intervention, changes in head position were measured. Results Scores on the Korean TIS remained unchanged, at 14/23 both before and after the intervention; scores on the PASS also remained unchanged, at $30 / 36$ both before and after the intervention. For static foot pressure, front foot pressure decreased by $7.3 \%$, from $55.4 \%$ before the intervention to $48.1 \%$ after the intervention, and back foot pressure increased by $7.3 \%$, from $44.6 \%$ to $51.9 \%$. In the 10 -meter walking test, the time taken to walk 10 meters decreased by 4 seconds, from 14 seconds to 10 seconds, and measurements by the GAITRite system showed that gait velocity decreased by 19.1 seconds, from 52.5 seconds to 33.4 seconds, and single foot support increased by 0.19 , from 0.09 to 0.28. Conclusion After 4 weeks of standing-up robot training and general physical therapy training, the 10-meter walking test and footprint showed improved results. This seems to be the result of improved standing and dynamic balancing ability of the patient with spastic diplegic cerebral palsy.
\end{abstract}

Key words Cerebral palsy, spastic diplegia, Tilt table stepping robot, Postural control, Balance

Corresponding author In-Tae Ryu(bobathryu@hanmail.net)
Received date
21 January 2019
Revised date
02 February 2019
Accepted date
17 February 2019

\section{Introduction}

Cerebral palsy is a developmental disorder of movement and posture that restricts different functional activities through non-progressive disturbances that occur during fetal or infant development. This motor disorder is accompanied by sensory, cognitive, communication, perceptual, and behavioral disabilities. ${ }^{1)}$ Children with cerebral palsy have enough force to balance, but the loss of sensory information that is unrecognized about the position of the balanced support surface due to asymmetry and the reduced ability to maintain the center of gravity between the hip

http:dx.doi.org/10.17817/2019.02.02.111371 joints on both sides makes it difficult to control the balance of stand of posture. ${ }^{2)}$ In addition, symptoms of cerebral palsy, such as joint deformations, muscle shortening, and visual and proprioceptive impairment can cause problems in balance performances such as posture maintenance timing and orientation of body and stride, which leads to hyper mobility, trunk instability, and difficulty with postural maintenance, affecting their standing and independent-gait ability. ${ }^{3), 4)}$

Due to lack of trunk stability and lower-limb mobility, children with cerebral palsy develop many compensatory strategies that take the form of balance control in other segments of the body-in particular the neck, upper extremities, and upper trunk. ${ }^{5)}$ When pa- 
tients with bilateral spastic cerebral palsy are in a standing posture, their feet are pronated; their lower legs are internally rotated; their knee joints are flexed; their hip joints are flexed, adducted, and internally rotated; and their pelvis is anteriorly tilted. (6),7) Such kinematic problems lead to a shift in the center of gravity in both sitting and standing postures and a decreased ability for gait and balance adjustment, resulting in bad posture. ${ }^{8)}$ Bobath asserted that cerebral palsy patients should be able to maintain a balanced standing posture and shift their weight onto their bilateral lower limbs to reduce asymmetry between the left and right sides of the body and to recover their gait ability.9) Woolacott reported that children with diplegic cerebral palsy and hemi cerebral palsy had impaired their ability to control their posture and walk.

Standing is the ability to spontaneously balance oneself against small postural sways. Maintenance of nervous system architecture and control of the motor system during standing necessitate body alignment to push the center against gravity, muscle tone to prevent the body from collapsing against gravity, and postural tone to enable action by antigravity muscles. ${ }^{10)}$ Notably, robot-based rehabilitation has been shown to improve motor performance by boosting brain plasticity. In particular, the ERIGO device(Hocoma AG, Volketswil, Switzerland) combines progressive verticalization, cyclic leg movement(which allows stepping reinforcement in combination with step synchronized muscle funcional electrical stimulation-FES- at lower limb), and body weight loading to ensure the safe stabilization of the parient in the upright position. ${ }^{32)}$ This study aims to examine the effects of standing-up robot rehabilitation in the context of a cerebral palsy patient's postural peculiarity and physical therapy based on the Bobath concept, with the aim of supporting her standing posture and gait ability.

\section{Materials and Methods}

\section{Subject and Study Period}

The subject was an 18-year-old female diagnosed with bilateral cerebral palsy and receiving ambulatory care at our hospital. She was able to clearly convey her needs and precisely understand the therapist's instructions. To measure her motor ability, the Gross Motor Function Measure was used; her total score was 75.56\% across five dimensions-lying and rolling (100\%), sitting (100\%), crawling and kneeling (78.57\%), standing (58.97\%), and walking, running, and jumping (40.28\%). The Modified Ashworth Scale-the primary clinical measure of muscle spasticity-was employed to evaluate her degree of spasticity. The bilateral lower limbs rated as G1, and her overall lower-limb muscle strength had weakened.

The study period was from August 6 to September 4, 2018. She received standing-robot rehabilitation training three times per week, 30 minutes each time, and general physical therapy three times per week, for four weeks. Erigo Pro(Hocoma) was used for her standing-up robot rehabilitation training. With her knees flexed at $90^{\circ}$, she received extensor muscle strengthening exercise together with training in sensory stimulation on the heels.

\section{Measurement Tools and Outcome Measures \\ (1) Korean Version of the Trunk Impairment Scale(K-TIS)}

The TIS consists of three sub-scales: static sitting balance, dynamic sitting balance, and coordination. The static sitting balance sub-scale evaluates three items-whether the patient maintains a sitting posture for 10 seconds without leaning or putting his or her hand on the floor (0-2 points), whether the patient maintains the posture when the examiner crosses the non-paretic leg over the paretic leg ( $0-2$ points), and whether compensatory movements occur in the upper extremity when the patient crosses the non-paretic leg over the paretic leg for himself or herself (0-3 points)-for a total score of $0-7$ points. The dynamic sitting balance sub-scale is composed of 10 items that examine the degree of selective lateral flexion of the upper and lower trunks, with 0-1 points scored for each item, for a total of $0-10$ points. The coordination sub-scale is composed of four items that measure selective rotation ability of the upper and lower trunks, 
with $0-1$ points or $0-2$ points scored for each item, for a total score of $0-6$ points. The TIS's three sub-scales have a total of 17 items, for a total score of 0-23 points.

Two physical therapists participated in measuring the reliability of the TIS when it was developed, during which the subjects were 28 acute and chronic stroke patients whose duration of stroke was 21 to 78 months. The inter-rater and test-retest reliabilities, expressed as intra-class correlation coefficients (ICCs), were 0.85-0.99 and 0.96-0.99, respectively. ${ }^{13)}$

\section{(2) Postural Assessment Scale for Stroke-Trunk Control (PASS)}

PASS was used to evaluate the subject's postural control and is composed of three basic postures (lying, sitting, standing) and 12 items (five items on postural maintenance ability and seven items on postural change), for a total of 36 points. Five items are used to measure trunk control. The four sub-domains of the items are sitting without assistance, rolling toward the less affected side from a supine position, sitting up on the corner of the bed from a supine position, and lying from a sitting position. The construct validity of PASS was found to be 0.73 , when compared to the Functional Independence Measure, its intra-rater reliability was 0.88 , and its test-retest reliability was $0.72{ }^{14)}$

\section{(3) Static Pressure on the Feet}

BIORescue (France) is a $610 \times 580 \times 10 \mathrm{~mm}$ force plate with 100 pressure sensors. The size of each pressure sensor is $10 \times 10 \mathrm{~m}$, each of which independently measures static and dynamic pressures of the feet in a standing posture or during movement. This study measured the static pressure of the subject's feet to examine the weight distribution of the feet in a standing posture. ${ }^{15}$ The evaluation of this study measured the foot pressure in a ten-second stand-up before and after intervention and measured the average value three times.

\section{(4) 10-Meter Walk Test}

The subject walked 10 meters, with the examiner starting timing when her toes passed the 2-meter mark and stopping timing when her toes passed the 8-meter mark. The examiner therefore measured the time taken for the subject to walk the intermediate 6 meters. ${ }^{16)}$ The evaluation of this study measured the average before and after intervention three times.

\section{(5) GAITRite}

A gait analyzer (GAITRite, CIR system Inc, USA, 2008) was used to evaluate the subject's gait ability. The patient's temporal and spatial gait ability was measured to quantitatively analyze her gait pattern (Figure 1). ${ }^{17), 18)}$ The gait analyzer is an electronic walkway whose length, width, and height were $5 \mathrm{~m}, 61 \mathrm{~cm}$, and $0.6 \mathrm{~cm}$, respectively, with 16,128 sensors of a diameter of $1 \mathrm{~cm}$ each of which was vertically arranged along the gait plate at intervals of $1.27 \mathrm{~mm}$ to collect data on temporal and spatial variables.

The data collected was processed using gait analysis software (GAITRite GOLD Version 3.2b, CIR system Inc, USA, 2007). The subject first stood in front of the gait plate, then walked at her most comfortable gait speed in response to the examiner's oral signal until leaving the walkway. Temporal gait characteristics, such as gait velocity, cadence, and spatial gait characteristics-including paretic side-step length, stride length, and single-limb support-were precisely measured through computer analysis. The test's intra-rater reliability was .90 and ICCs at a comfortable gait speed were 0.96 or higher. There was a high correlation with the measurements of gait characteristics using paper and a pencil $(\text { ICC }=.96)^{30)}$ and measurements using video motion analysis $($ ICC $=.94) .{ }^{17)}$

Temporal variables, such as stride time, supporting phase, and velocity may be used to evaluate problems with the subject's lower limbs. The system also quantifies gait patterns after gait training. ${ }^{17)}$ Spatial variables, such as step length time and gait length represent the lower limbs' degree of asymmetry and are therefore useful in many ways. ${ }^{21)}$ A study of stroke patients provided evidence of the reliability of the GAITRite system. The evaluation of this study measured the average before and after arbitration three times. 


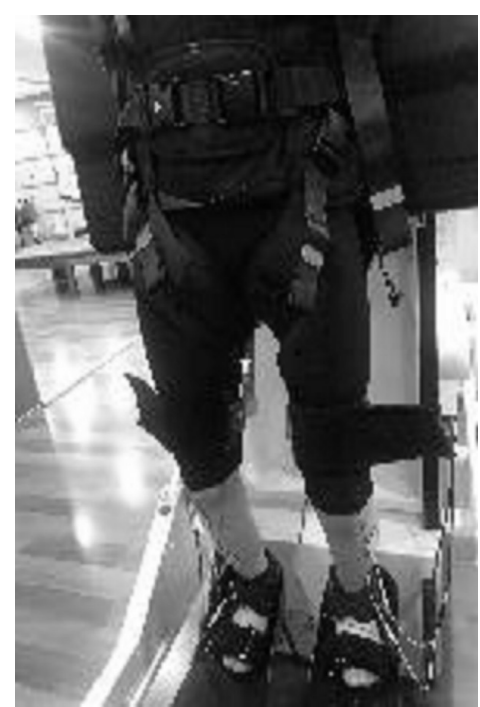

Figure 1-1. Tilt-table Stepping Robot Training (front)

\section{Intervention}

(1) Tilt-table Stepping Robot Training

The ERIGO device(Hocoma AG, Volketswil, Switzerland) combines progressive verticalization, cyclic leg movement(which allows stepping reinforcement in combination with step synchronized muscle funcional electrical stimulation-FES- at lower limb), and body weight loading to ensure the safe stabilization of the patient in the upright position. ${ }^{32)}$ Standing-up robot training triggers dynamic movements of the legs by applying regular weight loads to the lower limbs while changing their angles from $0^{\circ}$ to $90^{\circ}$ to the vertical on the foot plate with a spring. ${ }^{19), 20)}$

\section{(2) General Physical Therapy}

All interventions were neurodevelopmental treatments based on the Bobath concept, with the aim of improving effective movements and active participation in meaningful activities by maintaining proper body alignment and stabilizing each part of the body. The exercise program for strengthening lower-extremity ex-

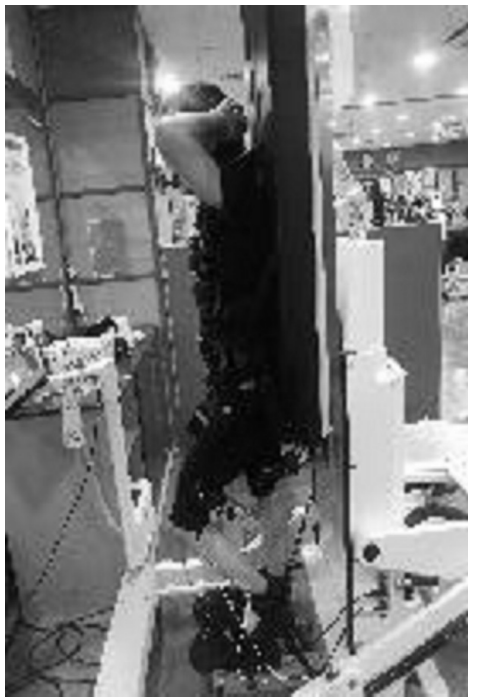

Figure 1-2. Tilt-table Stepping Robot Training(side)

tensors on the affected side focused on the hip extensors. ${ }^{11), 21)}$ The target extensors for this program were the hip abductor and knee extensor. The program was implemented in a supine position, side-lying position, and standing position seven times a week for 30 minutes each time over a four-week period.

Interventions were applied to the subject in a supine position, side-lying position, and standing position, three times per week, for 30 minutes. ${ }^{11,21)}$

\section{Results}

K-TIS remained unchanged, at 14/23 before and after the intervention; PASS also remained the same, at 30/36 before and after the intervention. For static foot pressure, front foot pressure decreased by 7.3\%, from $55.4 \%$ before the intervention to $48.1 \%$ after the intervention, and back foot pressure increased by $7.3 \%$, from $44.6 \%$ to $51.9 \%$. In the 10 -meter walking test, the

Table 1. Comparison of pre-training and post-training.

\begin{tabular}{ccccc}
\hline & & Pre-training & Post-training \\
\hline \multicolumn{2}{c}{ K-TIS } & $14 / 23$ & $14 / 23$ \\
\multirow{2}{*}{ GAITRSite } & PASW & $30 / 36$ & $30 / 36$ \\
& & 14 & 10 & 33.4 \\
& & velocity & 52.5 & 0.19 \\
\hline
\end{tabular}


Table 2. Comparison of footprints between pre-training and post-training.

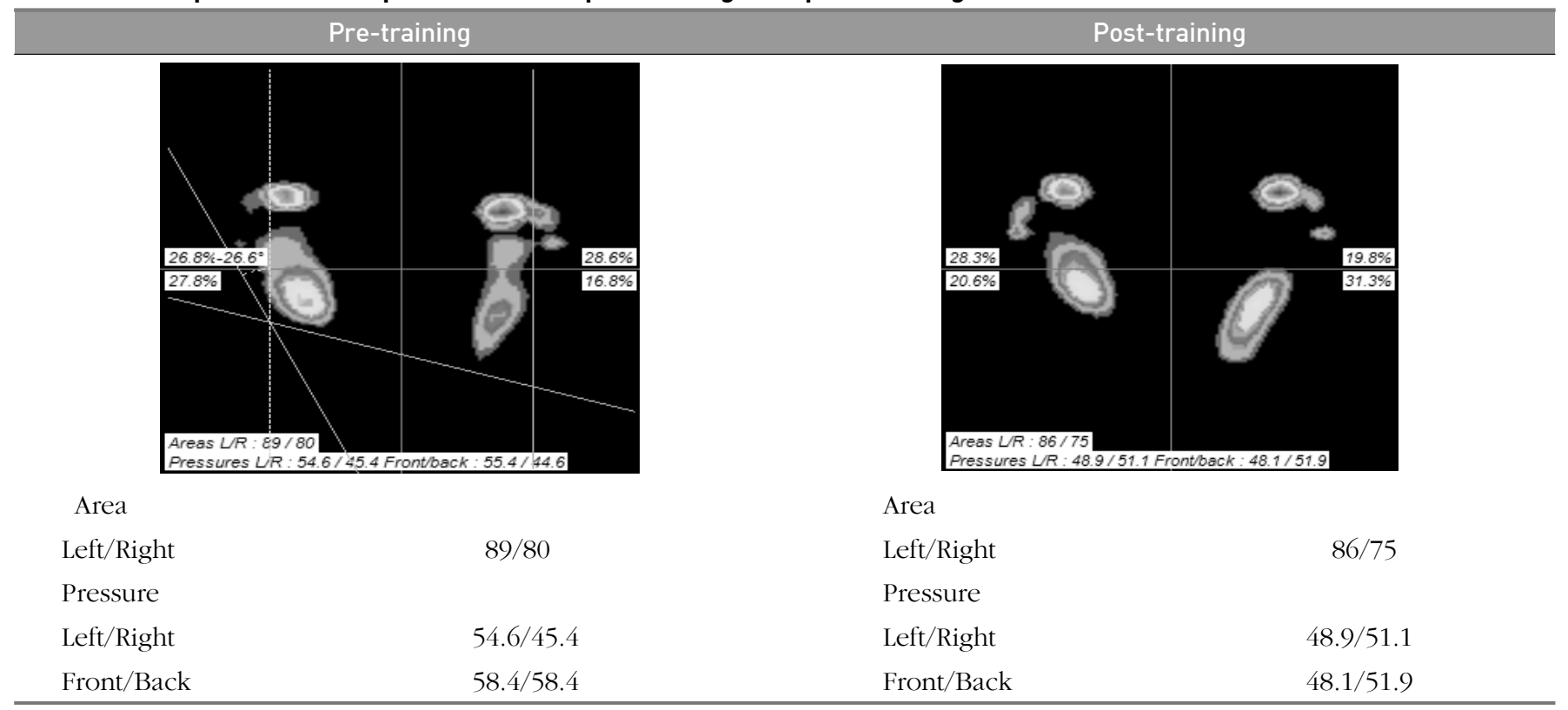

time taken to walk 10 meters decreased by 4 seconds, from 14 seconds to 10 seconds, and the measurements by GAITRite showed that gait speed decreased by 19.1 seconds, from 52.5 seconds to 33.4 seconds, and single support increased by 0.19 , from 0.09 to 0.28 .

\section{Discussion}

The ability of children with cerebral palsy to balance themselves in sitting and standing postures is important for quality of life through the activities of daily living. ${ }^{22}$ The biggest problems are an increase in abnormal shaking of the body and the shifting of weight onto the lower limb of the less affected side, which decrease the ability to balance during standing or walking. These characteristics of lower-limb support are the cause of patients' asymmetric standing posture. $^{23)}$

The corticospinal tract also makes it very difficult to control selective and voluntary movements; in particular, motor damage frequently occurs in the knee or ankle joints of the lower limbs. ${ }^{24)}$ Ankle movements assist in the connection between trunk muscles for postural control and are therefore very important. Hong $^{25)}$ noted that increased ankle movements enable control of lower-limb tone and increased voluntary movements, and, therefore, a program to move the ankles during treatment is necessary. In this study, in comparison before and after the foot pressure, the increase in the back foot pressure can be seen as an increase in the stability of the body in the standing position. $^{29), 30)}$

In general, posture is an essential element for normal balance, and refers to biomechanical alignment of human body parts or orientation of the body within an environment. ${ }^{26}$ Balance is the ability to maintain the center of the body within the basal surface and to continuously maintain body posture in response to environmental changes during body movements. Balance in a sitting or standing posture is an essential element for performance of body functions. ${ }^{27)}$ In spastic diplegic cerebral palsy, standing balance and gait ability have a closed relationship. ${ }^{28)}$

This study attempted to continuously control standing posture using sensory input information on the feet through standing-up robot training. Prior research has found that, while standing up, the body is stable when the pressure on the hindfoot is higher than on the forefoot, and the body is unstable when the pressure on the hindfoot is lower, and that a relative increase in the pressure on the hindfoot is a factor in 
controlling the stability of the body. This is in accordance with the results of the current study-increased weight distribution on the hindfoot, as measured by BIORescue, produced an increase in single stance according to GAITRite analysis. ${ }^{29), 30)}$ Arcan et al. ${ }^{31)}$ reported that exercise with weight load on the paretic lower limb and training on the shifting of weight onto the bilateral lower limbs increased balance control; training on the shifting of weight may be considered an initial developmental stage for gait, and even weight load on the bilateral lower limbs prevents secondary deformation and compensatory, abnormal patterns according to the physical disability.

This study measured the subject's footprint to examine changes in her weight distribution in a standing posture, gauged her weight distribution by foot pressure before and after the training, and observed an increase in her weight distribution This indicates an improvement in the subject's static standing-up balance ability. Further, the 10-meter walking test, which aimed to evaluate improvements in her gait function-which is a dynamic balance ability-showed that the time taken decreased after the training. These results support previous research that showed standing-up training is closely associated with dynamic balance ability and gait. ${ }^{29), 30)}$

\section{Conclusion}

This study was conducted to examine the effects of changes in standing postures on the gait ability of a cerebral palsy patient through standing-up robot training. After four weeks of standing-up robot training and general physical therapy based on the Bobath concept, the subject experienced improvements in a 10-meter walking test and footprint. This means that continuous standing-up robot training and physical therapy with a Bobath approach can be effective in improving a cerebral palsy patient's static and dynamic balance ability.

This study involved a specific patient as it was a case study. She had previously undergone other treatment activities, and therefore variables other than the interventions in this study were not controlled. Future research should address these limitations and examine the effects of standing-up training on the gait ability and postural change of a larger number of cerebral palsy patients in different age groups.

\section{References}

1. Bax M, Goldstein $\mathrm{M}$, Rosenbaum $\mathrm{P}$, et al. Proposeddefinition and classification of cerebral palsy. Dev Med Child Neurol. 2005;47:471-576.

2. Shumway-Cook, A., Anson, D. \& Haller, S(1988). Postural sway biofeedback: Its effect on restabilishing stance stability in hemiplegic patients. Arch Phys Med. Rehabili. 1988;69:395-400.

3. Donker SF, Ledebt A, Roerdink M, et al. Children with cerebral palsy exhibit greater and more regular postural sway than typically developing children. Exp Brain Res. 2008;184(3):363-70.

4. Gough M. Continuous postural managemental the prevention of deformity in children with cerebral palsy: an appraisal. Dev Med Child Neurol. 2009;51(2):105-110.

5. Bennett $\mathrm{C}$. The effectiveness of early intervention of infants at increased risk. Academic Press. 1987; 79-112.

6. Dakahashi. Clinical Movement Analysis. YeongMun publisher. 2000;351 -352.

7. Jordan R, Cusak J and Roseque B. Foot function and its relationship to postural in the pediatric patient with cerebral palsy and other notes and instroductional materials. Neurodevelopment Treatment Association, New York. 1983.

8. Stackhouse C, Shewokis PA, Pierce SR, et al. Gait initiation in children with cerebral palsy. Gait Posture. 2007;26(2):301-8.

9. Bobath B, Bobath K. Motor development in the different types of cerebral palsy. Butter worth-Heinemann Ltd, Oxford. 1984.

10. Shumway-Cook A. and Woollacott, MH. Motor Control: Theory and Practical Applications 2001;237-250.

11. Rain S. The current theoretical assumptions of the Bobath concept as determined by the members of BBTA. Physiotherapy Theory and Practice. 2007;23(3):137-157.

12. Russell D.J et al., Improved scaling of the gross motor function measure for children with cerebral palsy: Evidence of reliability and validity. Physical Therapy. 2000;80(9):873-885. 
13. Verheyden G, Kersten P. Investigating the internal validity of the trunk impairment scale using rasch analysis: the TIS 2.0 Disability and Rehabiliation. 2010;32(25):2127-37.

14. Benaim C, Perennou DA, Villy J, et al. Validation of a standardized assess-ment of postural control in stroke patients: the Postural Assessment Scale for Stroke Patients (PASS). Stroke. 1999;30(9):1862-8.

15. Lee J, Seo K. The effects of stair walking training on the balance ability of chronic stroke patients. J Phys Ther Sci. 20014;26(4):517-520.

16. Thompson P, Beath $\mathrm{T}$, Bell $\mathrm{J}$, et al. Test-retest reliability of the 10-metre fast walk test and 6-minute walk test in ambulatory school-aged children with cerebral palsy. Developmental Medicine \& Child Neurology. 2008;50(5):370-6.

17. McDonough, AL, Batavia M, et al. The validity and reliability of the GAIT Rite system's measurements: A preliminary evaluation. arch phys med rehabil. 2001;82(3): 419-425.

18. Van Uden CJ, \& Besser MP. (2004). Test-retest reliability of temporal and spatial gait character- istics measured with an instrumented walkway system(GAIT Rite). BMC musculoskelet disord. 2004;17(5):13.

19. Kressing, R.W., Gregor, R.J., Oliver, A., et al., Temporal and Spatial Features of Gait in Older Adults Transitioning to Frailty, Gait Postur. 2004;20(1):30-35.

20. Alexey N. Kuznetsov, NataliaV. Rybalko, VadimD.Daminov, et al. Early poststroke rehabilitation using a robotic tilt-table stepper and functional electrical stimulation. Stroke Research and Treatment. 2013.

21. Park HJ, (The) effects of strengthening exercise of paretic lower limb extensors on the cortico-reticulospinal tract in stroke. NEUROTHERAPY 2017;21(1):31-38.

22. Carlberg EB. And Hadders-Algra M. Postural dysfunction in Children with cerebral palsy: some implications for therapeutic guidance. Neural Plasticity. 2005;12(2-3): 221-228.
23. Bertoti DB. And Gross AL. Evaluation of biofeedback seat insert for improving active sitting posture in children sith cerebral palsy. Physical Therapy. 1988;68(7)1109-1113.

24. Fowler EG, Staudt LA, Greenberg MB. Lower-extremity selective voluntary motor control in patients with spastic cerebral palsy: increased distal motor impairment. Developmental Medicine and Child Neurology. 2010;52264(3):264-269.

25. Hong JS. Cerebral palsy treatment ideas. Seoul: koonja publishing company. 2011.

26. Cook AS. And Woollacott M. Motor Control: Translating Research into Clinical Practice. 3ed. Lippincott Williams and Wilkins. 2006;157-167.

27. Tyson SF, Connell LA. How to measure balance in clinical practice. A systematic review of the psychometrics and clinical utility of measure of balance activity for neurological conditions. Clin Rehabil. 2009;23(9): 824-40.

28. Liao HF, Jeng SF, Lai JS, et al. The relation between standing balance walking function in children with spastic diplegic cerebral palsy. Dev Med Child Neurol. 1997;9(2):106- 12.

29. Franco AH. Pes cavus and pes planus; analyses and treatment. Phys Ther. 1987;67(5)688-694.

30. Guskiewicz KM, Perrin DH. Reserch and clinica applications of assessing balance. J Sport Rehabil. 1996;5(3):45-63.

31. Arcan M, Brull MA, Najenson T, et al. Assessment of postural disorder during process of rehabilitation. Scand J Rehabil Med. 1997;9(4):165-68.

32. Rocco SC, Antonino N, Margherita R, et al. Do post-stroke patients benefit from robotic vercialization? A polot-study focusing on a novel neuroplysiological approach. Restorative Nerology and Neuroscience. 2015;(33): 678-681. 
